RESEARCH ARTICLE

\title{
Assessment of Pivka II as Tumour Marker in Hepatocellular Carcinoma
}

\author{
Anuj Parkash ${ }^{1 *}$, Parul Singla ${ }^{2}$ and Ashok Kumar Ahirwar ${ }^{3}$ \\ ${ }^{1}$ Department of Laboratory Medicine, Rajiv Gandhi Cancer Hospital and Research Centre, New Delhi \\ ${ }^{2}$ Department of Biochemistry, Sir Ganga Ram Hospital, New Delhi \\ ${ }^{3}$ Department of Biochemistry, University college of Medical Science, Delhi
}

*Corresponding author: Dr. Anuj Parkash, Department of Biochemistry, Rajiv Gandhi Cancer Institute \& Research Centre, Delhi, Tel: +919643001625

\begin{abstract}
Rationale: The most commonly used tumor marker in hepatocellular carcinoma (HCC) is serum alpha-fetoprotein (AFP). It has been routinely used for monitoring the prognosis of disease, but not for its detection. There is always a quest of new biomarkers for detection of HCC. The present study has been designed to determine the level of prothrombin induced by vitamin $\mathrm{K}$ absence-II (PIVKA II) in sera of patients suffering from $\mathrm{HCC}$.

Objectives: The aim of this study is to evaluate the PIVKA II as a biomarker for HCC independently or along with AFP to predict better sensitivity or specificity for diagnosing HCC.

Results: Using the best cut-off value of PIVKA II (37.5 $\mathrm{mAU} / \mathrm{ml}$ ) showed $94 \%$ sensitivity and $100 \%$ specificity for diagnosing hepatocellular carcinoma. While cut-off value of AFP $(6.5 \mathrm{ng} / \mathrm{ml})$, showed a sensitivity of $(79 \%)$ and specificity of $(100 \%)$ for diagnosing hepatocellular carcinoma.

Conclusions: In conclusion PIVKA II combined with AFP provided better results in terms of diagnostic accuracy and had a better sensitivity compared to either biomarker alone.
\end{abstract}

\section{Keywords}

PIVKA II, AFP, HCC

\section{Introduction}

Hepatocellular carcinoma (HCC) is considered to be the most common primary cancer of the liver. Liver cancer is the sixth most commonly diagnosed cancer and the fourth leading cause of cancer death worldwide in 2018 [1]. Age-standardised mortality rates (ASMR) from liver cancer in 2018 in both genders were highest in Ea- stern Asia (16.0) and lowest in South Central Asia (2.3). However by 2040 irrespective of sex and age group the estimated number of deaths from liver cancer increases by $64.3 \%$ [2]. The preventable common risk factors for HCC are Hepatitis B, Hepatitis C, liver cirrhosis and environmental toxins like aflatoxin, arecanut and contamination of ground water by industrial waste and use of various carcinogenic agricultural pesticide. Non alcoholic fatty liver, metabolic diseases, life style changes, and genetic factors are also known risk factors for hepatocellular carcinoma. Given its high incidence, with high mortality, an early diagnosis is an important imperative while countering this cancer [3]. The diagnosis of HCC includes serum markers, different imaging techniques and histological analysis.

The most commonly used tumor marker in HCC is AFP. Serum alpha-fetoprotein (AFP) has been routinely used as a tumor biomarker for monitoring the prognosis of disease [4]. Therefore, there is need of new biomarkers for detection of HCC. Various authors are studying PIVKA as an emerging tumor marker used independently or in conjunction with AFP for evaluating their role in HCC. This is a Protein induced by vitamin $\mathrm{K}$ absence (PIVKA-II) [5]. The aim of this study was to evaluate the PIVKA-II as a new biomarker for HCC independently or along with AFP both to predict a better sensitivity for diagnosing HCC.

\section{Material and Method}

This study was conducted in department of Labora- 
tory medicine of specialized cancer hospital. A total of 35 patients of HCC and 5 healthy controls were enrolled over a period of 1 year. Demographic and clinical details of the patients were recorded. Consent was taken from all the patients as per declaration of Helsinki. In control group history of any cancer or inflammatory disease were excluded.

Patients with any history of haemocoagulatory disorders, vitamin $\mathrm{K}$ uptake disorders, intake of vitamin $\mathrm{K}$ blocking agents, and cancer other than liver, acute inflammatory disease, renal and liver failure were excluded from the study. All the cases who were clinically suspected cases of hepatocellular carcinoma (HCC) and undergoing the diagnostic evaluation for HCC were enrolled in the study.

Blood sample was collected as per CLSI guidelines for measuring Liver function test, AFP and PIVKA. Serum was separated by centrifugation at $1,300 \times \mathrm{g}$ for $10 \mathrm{~min}$.

An assay of PIVKA was measured using a commercially available chemiluminescent assay of the Abbott Architect 1000i System (Abbott, Libertyville, IL, USA) and AFP was measured using a commercially available electro-chemiluminescent immunoassay (ECLIA) test kits from Ortho Clinical diagnostics through the VITROS 5600 equipment (Ortho Clinical diagnostics, US) . Other laboratory tests of Liver function tests (LFT), Renal function tests (RFT) were performed using commercial available test kits of Beckman Coulter on Beckman Coulter $\mathrm{AU} 480$ equipment (Beckman Coulter, US) to rule out the presence of clinical conditions that may affect the serum level of AFP and PIVKA.

\section{Statistical analysis}

The data was analysed by appropriate statistical methods using Statistical Package for Social Sciences (SPSS) $20^{\text {th }}$ Edition, IBM, NY, USA and Graph pad, USA. All the data were expressed as mean \pm SD and median and interquartile range if appropriate. Chi square test was used to analyse categorical variables. The student's t-test was used to compare the mean in two groups in parametric distribution of data. Mann Whitney $U$ test was used to compare the mean in two groups for nonparametric data. The Pearson's correlation analysis was performed to find strength and direction of relation among study variables. ROC curve was used to compare test performance and establishing cut off of serum PIVKA II and AFP levels in diagnosing hepatocellular carcinoma. The $p$ value of $<0.05$ was considered as statistically significant.

\section{Results}

\section{Demographic and Biochemical characteristics of the subjects in study population}

The demographical and biochemical characteristics of study population are shown in Table 1 and Table 2 . The present study was age and sex matched with control group.

\section{Serum PIVKA II and AFP in study population}

As shown in Table 3 the mean level of Serum PIVKA

Table 1: Demographic profile of study population.

\begin{tabular}{|l|l|l|l|l|}
\hline S.No & Parameters & Case $(\mathbf{n}=\mathbf{3 5})$ & Control $(\mathbf{n}=\mathbf{5})$ & P value \\
\hline 1 & Age (year) & $58 \pm 12$ & $65 \pm 8$ & 0.145 \\
\hline 2 & Sex (Male/Female) & $31 / 4$ & $5 / 0$ & 0.426 \\
\hline
\end{tabular}

Table 2: Biochemical parameters of study population.

\begin{tabular}{|l|l|l|l|l|}
\hline S.No & & $\begin{array}{l}\text { Case }(\mathbf{n}=\mathbf{3 5}) \\
\text { Median }(\text { Interquartile range) }\end{array}$ & $\begin{array}{l}\text { Control }(\mathbf{n}=\mathbf{5}) \\
\text { Median (Interquartile range) }\end{array}$ & P value \\
\hline 1 & Parameters & $0.9(0.7-1.6)$ & $0.6(0.5-0.6)$ & $0.030^{*}$ \\
\hline 2 & & $0.3(0.2-0.6)$ & $0.1(0.1-0.2)$ & $0.012^{*}$ \\
\hline 3 & & $47(29-85)$ & $43(27-76)$ & 0.737 \\
\hline 4 & AST (IU/L) & $55(39-81)$ & $31(25-58)$ & 0.061 \\
\hline 5 & Direct Bilirubin $(\mathrm{mg} / \mathrm{dl})$ & $135(88-199)$ & $111(60-142)$ & 0.120 \\
\hline 6 & ALT (IU/L) & $93(62-132)$ & $80(47-134)$ & 0.462 \\
\hline
\end{tabular}

* $p$ value $\leq 0.05$ is considered statistically significant. ALT: Alanine Transaminase; AST: Aspartate Transaminase; ALP: Alkaline Phosphatase; GGT: Gamma Glutamyl Transferase

Table 3: Serum PIVKA level in Study Population.

\begin{tabular}{|l|l|l|l|}
\hline Parameters & $\begin{array}{l}\text { Cases }(\mathbf{n}=\mathbf{3 5}) \\
\text { Median }(\text { Interquartile range) }\end{array}$ & $\begin{array}{l}\text { Controls }(\mathbf{n}=\mathbf{5}) \\
\text { Median (Interquartile range) }\end{array}$ & P value \\
\hline PIVKA-II & $1787(96-5137)$ & $23(20-31)$ & $0.000^{*}$ \\
\hline AFP & $19(7-152)$ & $4(4-6)$ & $0.004^{*}$ \\
\hline
\end{tabular}

${ }^{*} p$ value $\leq 0.05$ is considered statistically significant. 
Table 4: Correlation analysis of serum PIVKA with study variables.

\begin{tabular}{|l|l|l|l|l|l|}
\hline S. No. & Parameters & PIVKA II & \multicolumn{2}{l|}{ AFP } \\
\cline { 3 - 6 } & & r value & p value & r value & p value \\
\hline 1 & PIVKA II & 1 & - & 0.428 & $0.007^{*}$ \\
\hline 2 & AFP & 0.428 & $0.007^{*}$ & - \\
\hline 3 & Total Bilirubin & 0.386 & $0.015^{*}$ & 0.373 & $0.019^{*}$ \\
\hline 4 & Direct Bilirubin & 0.392 & $0.014^{*}$ & 0.375 & $0.019^{*}$ \\
\hline 5 & ALT & -0.094 & 0.569 & -0.068 & 0.679 \\
\hline 6 & AST & 0.068 & 0.680 & -0.002 & 0.992 \\
\hline 7 & ALP & 0.346 & $0.031^{*}$ & 0.076 & 0.647 \\
\hline 8 & GGT & 0.151 & 0.360 & -0.036 & 0.829 \\
\hline
\end{tabular}

" $p$ value $\leq 0.05$ is considered statistically significant. $r=$ Pearson's correlation coefficient

FBG: Fasting Blood Glucose; ALT: Alanine Transaminase; AST: Aspartate Transaminase; ALP: Alkaline Phosphatase; GGT: Gamma Glutamyl Transferase

Table 5: Area under ROC curve of PIVKA II and AFP in study population.

\begin{tabular}{|l|l|l|l|l|}
\hline S. No & Parameters & Area Under ROC curve & P Value & 95 \% Confidence Interval \\
\hline 1 & PIVKA II & 0.98 & 0.000 & $0.95-1$ \\
\hline 2 & AFP & 0.87 & 0.007 & $0.768-0.991$ \\
\hline
\end{tabular}

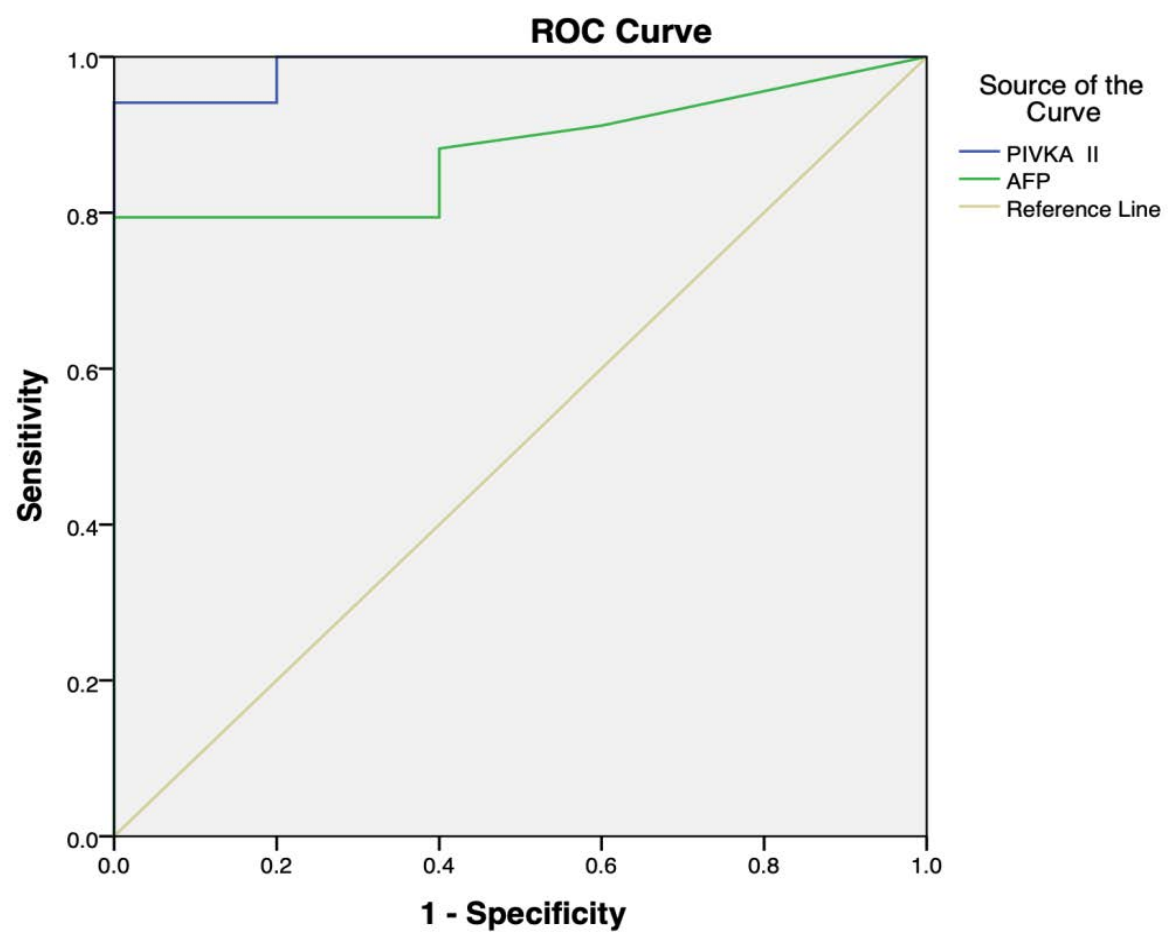

Figure 1: ROC curve for estimating cut-off of serum PIVKA II and AFP levels for diagnosing hepatocellular carcinoma. According to this graph that at cut-off of 37.5 , the serum PIVKA II has $94 \%$ sensitivity and $100 \%$ specificity for diagnosing hepatocellular carcinoma and at cut-off of 6.5 , the serum AFP has $79 \%$ sensitivity and $100 \%$ specificity for diagnosing hepatocellular carcinoma.

II and AFP was significantly higher in cases as compared to controls. Table 4 shows correlation analysis of Serum PIVKA II and AFP with biochemical parameters.

\section{ROC curve was used to compare tests by area un- der curve (AUC)}

As shown in Figure 1, Table 5, PIVKA II covers $98 \%$ area under ROC as compared to AFP, which covers $87 \%$ area under ROC. This denotes that serum PIVKA II is better test than serum AFP in predicting hepatocellular carcinoma. According to this ROC curve, that at cut-off of 37.5 , the serum PIVKA II has $94 \%$ sensitivity and $100 \%$ specificity for diagnosing hepatocellular carcinoma and at cut-off of 6.5 , the serum AFP has $79 \%$ sensitivity and $100 \%$ specificity for diagnosing hepatocellular carcinoma as shown in Table 6. 
Table 6: Sensitivity and specificity of PIVKA II and AFP in predicting hepatocellular carcinoma.

\begin{tabular}{|l|l|l|}
\hline Diagnostic Parameters & Sensitivity & Specificity \\
\hline PIVKA (cut-off 37.5) & $94 \%$ & $100 \%$ \\
\hline AFP (cut-off 6.5 ) & $79 \%$ & $100 \%$ \\
\hline
\end{tabular}

\section{Discussion}

In India hepatocellular carcinoma (HCC) is the $6^{\text {th }}$ most common cause of death in both males and females [6]. In our study males are found to be affected more than females but $50 \%$ cases are in age group of $50-70$ years. Our results are synonymous with other study which states HCC is commonly present in middle age man. The most probable independent risk factor associated with $\mathrm{HCC}$ in males is heavy drinking and smoking [7].

Tumour markers are used in routine to assess the prognosis of cancer treatment. The tumour marker used in clinical practice to support diagnosis of HCC is AFP. In the present study sensitivity of AFP at cut off 6.5 was observed $79 \%$. Hu, et al. in their study observed at cut off $20 \mathrm{ng} / \mathrm{mL}$ the sensitivity of AFP is $60 \%$ [8]. In another observational study by Nguyen-Dinh, et al. on 24000 cases of HCC seen over a 7-year period at a tertiary referral hospital of Vietnam the tumor marker AFP was raised only in approximately half of the cases which question marks the use of this biomarker alone in screening of their patients [9]. AFP is an oncofetal antigen, were found to be increased in various other malignant conditions other than HCC and chronic liver diseases [10]. So there is always a quest to find new marker of HCC. Protein induced by vitamin K absence-II (PIVKA-II) - also known as des- $\nu$-carboxy-prothrombin-has been identified as a serum biomarker linked to HCC. PIVKA or DCP is an abnormal form of coagulation protein prothrombin. It is induced by the absence of vitamin $\mathrm{K}$ [5]. In the present study the median levels of PIVKA are more than median levels of AFP. The AUROC for PIVKA is 0.98 and for AFP is 0.87 . The sensitivity observed of PIVKA at cut off $37.5 \mathrm{mAU} / \mathrm{mL}$ in case group is $94 \%$. Seo, et al. studied during differentiation of HCC from nonmalignant $\mathrm{CHB}$ at cutoff values of $40 \mathrm{mAU} / \mathrm{mL}$ for PIVKA-II, AUROC 0.854 sensitivity $73.9 \%$ [11]. Wang, et al. in their study on diagnostic utility of PIVKA-II in early stage HBV related HCC found ROC analysis for serum PIVKA-II level was depicting better sensitivity and specificity as compared to Non HCC subjects. The cause for high PIVKA-II could be deranged liver function which causes hyperbilirubenemia and inturn lead to malfunction of vitamin $\mathrm{K}$ absorbance and induce PIVKA production. Further they study higher PIVKA-II level in poorly differentiated-undifferentiated group, microvascular invasion group. It could be due to proliferation and migration of human umbilical vein endothelial cells by PIVKA [12]. This promotes production of vascular endothelial growth factor in hepatoma cell lines and enhances angiogenesis. Baek, et al. demonstrated in their study that irrespective of the diameter of HCC the diagnostic accuracy of PIVKA was higher than that of AFP [13]. Several studies from Asian countries have shown that serum PIVKA-II levels correlate with the HCC stage, as well as with survival of HCC patients [14-16]. The level of PIVKA is closely associated with a larger tumor, vascular invasion and it serves as a more accurate tumor marker compared with AFP $[17,18]$. It has been observed if AFP was combined with PIVKA the sensitivity to diagnose $\mathrm{HCC}$ will improve and also predict the recurrence of $\mathrm{HCC}$ within 6 months after surgery [19]. In Japan the recommended Clinical Practice Guidelines for diagnosis and surveillance of HCC uses combination of PIVKA-II and AFP [20].

This study has some limitations. The sample size is small in study. Also the study groups in material and method did not differentiate HCC patients on the basis of cirrhosis or histopathological grading. The changes in PIVKA-II during treatment of patient may provide more information on prognostic value of the biomarker. So, follow-up study with relatively large sample size is required.

In conclusion PIVKA-II combined with AFP provided better results in terms of diagnostic accuracy and had a better sensitivity and specificity compared to either biomarker alone.

\section{Sources of Support}

Nil.

\section{Author Contributions}

All the authors contributed equally by designing the research study, collecting the samples, conducting the experiments, acquiring the data, statistical analysis and writing the manuscript and no conflict of interest.

\section{References}

1. Bray F, Ferlay J, Soerjomataram I, Siegel RL, Torre LA, et al. (2018) Global Cancer Statistics 2018: GLOBOCAN Estimates of Incidence and Mortality Worldwide for 36 Cancers in 185 Countries. CA: A Cancer Journal for Clinicians 68: 394-424.

2. Rawla1 P, Sunkara T, Muralidharan P, Raj JP (2018) Update in global trends and aetiologyof hepatocellular carcinoma. Contemp Oncol (Pozn) 22: 141-150.

3. Kew MC (2014) Hepatocellular carcinoma: Epidemiology and risk factors. Journal of Hepatocellular Carcinoma 1: 115-125.

4. Gomaa Al, Khan SA, Leen ELS, Waked I, Taylor-Robinson SD (2009) Diagnosis of hepatocellular carcinoma. World J Gastroenterol 15: 1301-1314.

5. Weitz IC, Liebman HA (1993) Des-y -carboxy (Abnormal) Prothrombin and Hepatocellular Carcinoma: A Critical Review. Hepatology 18: 990-997.

6. Omata M, Cheng AL, Kokuda N, Kudo M, Lee JM, et al. (2017) Asia-Pacific clinical practice guidelines on the management of hepatocellular carcinoma: A 2017 update. Hepatology International 11: 317-370.

7. Purohit V, Rapaka R, Kwon OS, Song BJ (2013) Roles of 
alcohol and tobacco exposure in the development of hepatocellular carcinoma. Life Sci 92: 3-9.

8. Hu J, Wang N, Yang Y, Ma L, Han R, et al. (2018) Diagnostic value of alpha-fetoprotein combined with neutrophil-to-lymphocyte ratio for hepatocellular carcinoma. BMC Gastroenterology 18: 186.

9. Nguyen-Dinh SH, Do A, Doan Pham TN, Dao DY, Nguy TN, et al. (2018) High burden of hepatocellular carcinoma and viral hepatitis in Southern and Central Vietnam: Experience of a large tertiary referral center, 2010 to 2016 . WorId J Hepatol 10: 116-123.

10. Alpert E, Feller ER (1978) Alpha feto protein in benign liver disease- Evidence that normal liver regeneration does not induce AFP synthesis. Gastroenterology 74: 756-758.

11. Seo SI, Kim HS, Kim WJ, Shin WG, Kim DJ, et al. (2015) Diagnostic value of PIVKA-II and alpha-fetoprotein in hepatitis B virus associated hepatocellular carcinoma. World J Gastroenterol 21: 3928-3935.

12. Wang X, Zhang W, Liu Y, Gong W, Sun P, et al. (2017) Diagnostic value of prothrombin induced by the absence of vitamin K or antagonist-II (PIVKA-II) for early stage HBV related hepatocellular carcinoma. Infectious Agents and Cancer 12: 47.

13. Baek YK, Lee JH, Jang JS, Lee SW, Han JY, et al. (2009) Diagnostic role and correlation with staging systems of $\mathrm{PI}$ VKA-II compared with AFP. Hepatogastroenterology 56: 763-767.
14. Imamura $H$, Matsuyama $Y$, Miyagawa $Y$, Ishida $K$, Shimada $R$, et al. (1999) Prognostic significance of anatomical resection and des-gamma-carboxy prothrombin in patients with hepatocellular carcinoma. Br J Surg 89: 1032-1038.

15. Nakamura S, Nouso K, Sakaguchi K, Ito YM, Ohashi Y, et al. (2006) Sensitivity and specificity of des-gammacarboxy prothrombin for diagnosis of patients with hepatocellular carcinomas varies according to tumor size. Am J Gastroenterol 101: 2038-2043.

16. Saitta C, Raffa G, Alibrandi A, Brancatelli S, Lombardo D, et al. (2017) PIVKA-II is a useful tool for diagnostic characterization of ultrasound-detected liver nodules in cirrhotic patients. Medicine 96: 26.

17. Yamamoto $K$, Imamura $H$, Matsuyama $Y$, Kume $Y$, Ikeda $\mathrm{H}$, et al. (2010) AFP, AFP-L3, DCP, and GP73 as markers for monitoring treatment response and recurrence and as surrogate markers of clinicopathological variables of HCC. J Gastroenterol 45: 1272-1282.

18. Koike $\mathrm{Y}$, Shiratori $\mathrm{Y}$, Obi S, Teratani T, Imamura, et al. (2001) Des-gamma-carboxy prothrombin as a useful predisposing factor for the development of portal venous invasion in patients with hepatocellular carcinoma: A prospective analysis of 227 patients. Cancer 91: 561-569.

19. Ertle JM, Heider D, Wichert M, Keller B, Kueper R, et al. (2013) A combination of alpha-fetoprotein and des-gamma-carboxy prothrombin is superior in detection of hepatocelullar carcinoma. Digestion 87: 121-131.

20. M Kudo (2015) Clinical Practice Guidelines for Hepatocellular Carcinoma Differ between Japan, United States, and Europe. Liver Cancer 4: 85-95. 